\title{
Learning to recognize visual stimuli in neuromorphic VLSI
}

\author{
Federico Corradi \\ Institute of Neuroinformatics (INI), \\ University of Zurich and ETH, \\ Zurich, Switzerland \\ federico@ini.phys.ethz.ch
}

\author{
Massimiliano Giulioni \\ Technology and Health department \\ Istituto Superiore di Sanità (ISS) \\ Rome, Italy \\ Massimiliano.Giulioni@iss.infn.it
}

\begin{abstract}
We demonstrate learning in a neuromorphic recurrent attractor network distributed onto two VLSI chips. On a monitor we present some stimuli which are input to the network through the neuromorphic retina. Stimulation induces modification in the synaptic weights up to the point in which the selective reverberant states of activity are supported in the absence of stimulus. The network activity and the evolution of the synaptic matrix are monitored during learning. The visitor can draw his/her own stimuli, he/she can modify the learning parameters to teach the network to recognize them.
\end{abstract}

\section{SETUP}

The setup includes a monitor to display the stimuli, a neuromorphic retina [1] to acquire them and a VLSI neuromorphic network that learns in an unsupervised fashion. The devices are connected using a PCI-AER board which connects chips and retina and communicates with the PC. The pattern of synaptic connectivity is fixed. The PC is just for monitoring the activity and it does not affect the network dynamics.

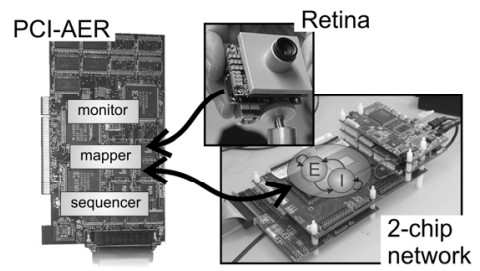

Every chip hosts 128 integrate-and-fire neurons and 16384 hebbian plastic stochastic bystable synapses. The network is fully reconfigurable: for this demo we use an inhibitory population of 50 neurons plus an excitatory population of 200 neurons. Throughout learning the initial homogeneous network tends to create clusters of neurons (one per input stimulus) that, at the end of learning reaches a sufficiently high level of positive feedback to self-sustain their activity even after the release of the stimulus.

\section{VISITOR EXPERIENCE}

The role of the visitor is to play with our setup to lead the network to learn a pair of visual stimuli. With a user-friendly interface the visitors draw a couple of stimuli, choose a learning protocol and observe the network evolution during learning. The state of the network is shown every few seconds thus visualizing how learning proceeds and how it is affected by various parameters. After learning, in a test phase the visitor can corrupt the original stimuli to test the error correction ability of this attractor network.

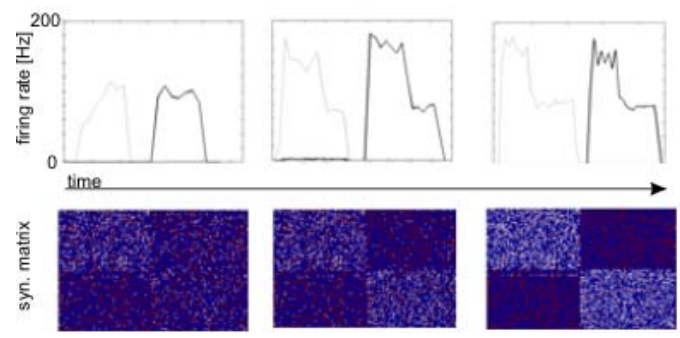

The demo has been thought to show the robustness of this kind of attractor architecture [2,3] to various sources of noise: from the circuitry mismatch to the input noise. A fault-tolerant architecture which can reliably learn, in an unsupervised fashion, to recognize visual stimuli.

[1] Lichsteiner, P. , P. C. Posch, T. Delburck. "A 128x128db 15us Latency Asynchronous Temporal Contrast Vision Sensor" IEEE Journal of Solid State Circuits, Feb 2008 43(2) 566-576. http://www.ini.uzh.ch/ tobi/wiki/lib/exe/fetch.php?media=lichtsteiner dvs jssc08.pdf

[2] P. Del Giudice, S. Fusi and M. Mattia, "Modeling the formation of working memory with networks of integrate-and-fire neurons connected by plastic synapses" Journal of Physiology Paris 97 2003) pp. $659-681$ http://neural.iss.infn.it/Papers/DelGiudiceFusiMattia_JPhysiolParis.pd $\underline{\mathrm{f}}$

[3] M. Giulioni, P. Camilleri, M. Mattia, V. Dante, J. Braun, and P. Del Giudice, "Robust working memory in an asynchronously spiking neural network realized with neuromorphic VLSI", Frontiers in Neuromorphic Engineering 5:149. doi: 10.3389/fnins.2011.00149 http://neural.iss.infn.it/Papers/Giulioni_et_al_Frontiers.pdf 